\title{
Low to High Spin-State Transition Induced by Charge Ordering in Antiferromagnetic $\mathrm{YBaCo}_{2} \mathrm{O}_{5}$
}

\author{
T. Vogt, ${ }^{1}$ P. M. Woodward, ${ }^{2}$ P. Karen, ${ }^{3}$ B. A. Hunter, ${ }^{4}$ P. Henning, ${ }^{1}$ and A. R. Moodenbaugh ${ }^{5}$ \\ ${ }^{1}$ Physics Department, Brookhaven National Laboratory, Upton, New York 11973-5000 \\ ${ }^{2}$ Department of Chemistry, The Ohio State University, 100 West 18th Avenue, Columbus, Ohio 43210-1185 \\ ${ }^{3}$ Department of Chemistry, University of Oslo, P.O. Box 1033, Blindern, 0315 Oslo, Norway \\ ${ }^{4}$ Australian Nuclear Science \& Technology Organization, Private Mail Bag 1, Menai, NSW 2234, Australia \\ ${ }^{5}$ Department of Applied Sciences, Brookhaven National Laboratory, Upton, New York 11973-5000
}

(Received 4 August 1999)

\begin{abstract}
The oxygen-deficient double perovskite $\mathrm{YBaCo}_{2} \mathrm{O}_{5}$, containing corner-linked $\mathrm{CoO}_{5}$ square pyramids as principal building units, undergoes a paramagnetic to antiferromagnetic spin ordering at $330 \mathrm{~K}$. This is accompanied by a tetragonal to orthorhombic distortion. Below $220 \mathrm{~K}$ orbital ordering and long-range $\mathrm{Co}^{2+} / \mathrm{Co}^{3+}$ charge ordering occur as well as a change in the $\mathrm{Co}^{2+}$ spin state from low to high spin. This transition is shown to be very sensitive to the oxygen content of the sample. To our knowledge this is the first observation of a spin-state transition induced by long-range orbital and charge ordering.
\end{abstract}

PACS numbers: 75.50.Ee, 61.12.-q

The recent discovery of colossal magnetoresistance in manganese oxides has sparked a renaissance in the study of spin, charge, and orbital ordering in transition metal oxides. A related phenomenon, not observed in the manganates due to strong Hund's rule coupling, is a spin-state transition. These are observed in the isostructural cobaltates, such as $\mathrm{LaCoO}_{3}$, which has a low spin ${ }^{6} t_{2 g}{ }^{0} e_{g}$ magnetic ground state. On warming a spin-state transition to an intermediate ${ }^{5} t_{2 g}{ }^{1} e_{g}$ spin state is observed near $100 \mathrm{~K}$ $[1,2]$. At $500 \mathrm{~K}$ a second spin-state transition involving high and low spin $\mathrm{Co}^{3+}$ occurs. The specifics of these thermally activated low to high spin-state transitions are still controversial [3,4]. They have also been shown to prevail in dilute hole-doped systems such as $\mathrm{La}_{1-x} \mathrm{Sr}_{x} \mathrm{CoO}_{3}$ [5]. We report here on structurally related $L \mathrm{BaCo}_{2} \mathrm{O}_{5+x}$ materials, whose structures are derived from perovskites via ordering of the rare earth $(L)$ and $\mathrm{Ba}$ cations into layers along $c$ and removing oxygen exclusively from the $L$ layer [6,7]. This creates an apically connected double layer of corner-sharing $\mathrm{CoO}_{5}$ pyramids. For $x>0$ the extra oxygen ions are incorporated into the $L$ layer of $L \mathrm{BaCo}_{2} \mathrm{O}_{5+x}$ to form disordered octahedra along the $c$ axis. We have synthesized $L \mathrm{BaCo}_{2} \mathrm{O}_{5.00}$ and studied the thermal evolution of its structure and properties using synchrotron $\mathrm{x}$-ray [8] and neutron powder diffraction [9] as well as conductivity and magnetization measurements. This composition was selected since equal concentrations of $\mathrm{Co}^{2+}$ and $\mathrm{Co}^{3+}$ should maximize the Coulomb stabilization energy of the charge-ordered state. The samples were synthesized from nanoscale precursors obtained by liquid mixing of citrate melts [10]. They were single phase according to conventional powder $\mathrm{x}$-ray diffraction measurements. Highresolution synchrotron $\mathrm{X}$-ray powder diffraction at $300 \mathrm{~K}$ confirmed this for the $L=$ Gd sample, whereas for $L=\mathrm{Y}$ approximately $10 \%$ of the same phase with slightly enhanced oxygen content was detected. This minority phase is thought to be a result of surface oxidation during storage at room temperature. The results we report below clearly demonstrate the occurrence of orbital and charge ordering, accompanied by a low to high spin-state transition upon cooling.

Above $330 \mathrm{~K} \mathrm{YBaCo} \mathrm{O}_{5}$ is paramagnetic and tetragonal (space group $P 4 / \mathrm{mmm}$ ). At approximately $330 \mathrm{~K}$ an antiferromagnetic (AFM) phase transition occurs as indicated by a cusp in the magnetic susceptibility measurements (Fig. 1) and the appearance of magnetic superstructure reflections in neutron powder diffraction data. Synchrotron x-ray powder diffraction measurements show that this magnetic transition occurs simultaneously with a tetragonal-to-orthorhombic $(T-O)$ structural phase transition. Furthermore, as mentioned above, the minority tetragonal phase persists below the $T-O$ transition. The two-phase coexistence was confirmed by ultra-highresolution diffraction measurements using a crystal analyzer. We stress that the observation of two phases in synchrotron x-ray powder diffraction experiments, with an order of magnitude better resolution than available with a standard laboratory x-ray diffractometer, can be traced to minute oxygen-content variations in the order of magnitude around $x=0.01$ [11]. In the presence of strong lattice coupling these small compositional variations can lead to phase coexistence, which can be observed in high-resolution experiments. This behavior beautifully illustrates how sensitive phase transitions within these systems are to minute variations in the oxygen stoichiometry.

At room temperature there is no evidence for any long-range charge ordering. However, upon cooling long-range charge ordering is detected below $200 \mathrm{~K}$, by the appearance of the $\left(\frac{1}{2} 02\right),\left(\frac{1}{2} 12\right),\left(\frac{1}{2} 10\right)$, and $\left(\frac{1}{2} 11\right)$ superlattice reflections indicating a doubling of the unit cell along the $a$ axis. These very weak superlattice reflections, 

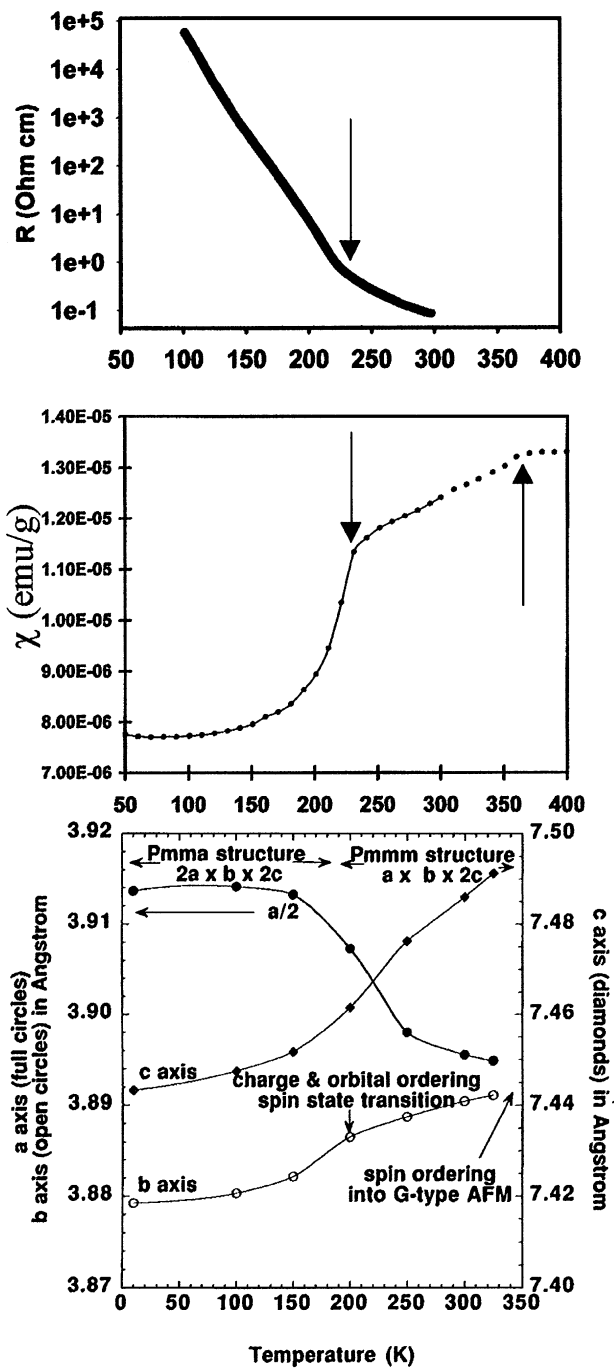

FIG. 1. Four-probe resistivity and magnetization data of $\mathrm{YBaCo}_{2} \mathrm{O}_{5}$ data indicate two phase transitions (arrows) near 350 and $220 \mathrm{~K}$. In the lower panel the evolution of the lattice parameters as a function of temperature is shown. For details, see text.

which have intensities of $\sim 0.1 \%$ of the strongest Bragg reflection were observed in both the position sensitive detector (PSD) and the $\mathrm{Si}(111)$ crystal analyzer modes. In order to explain the presence of these superlattice reflections we considered the following structural phase transformation mechanisms: (a) rotations of the square pyramidal units about [001], $\mathrm{Co}^{2+} / \mathrm{Co}^{3+}$ charge ordering into (b) a rocksalt arrangement, similar to that observed in $\mathrm{YBaMn}_{2} \mathrm{O}_{5}$ [12], (c) checkerboard ordering in the $a b$ plane, inverted in the $c$ direction, similar to $\left(\mathrm{Nd}_{0.5} \mathrm{Sr}_{0.5}\right) \mathrm{MnO}_{3}$ [11], (d) chains of $\mathrm{Co}^{2+}$ ions running parallel to [010] but alternating with $\mathrm{Co}^{3+}$ chains in the $a$ and $c$ directions (see Fig. 2). Table I plainly shows that only model (d) is consistent with the observed unit cell and space group. Figure 1 shows the evolution of the unit cell dimensions, magnetic susceptibility, and electrical resistivity with temperature. Near $220 \mathrm{~K}$ we
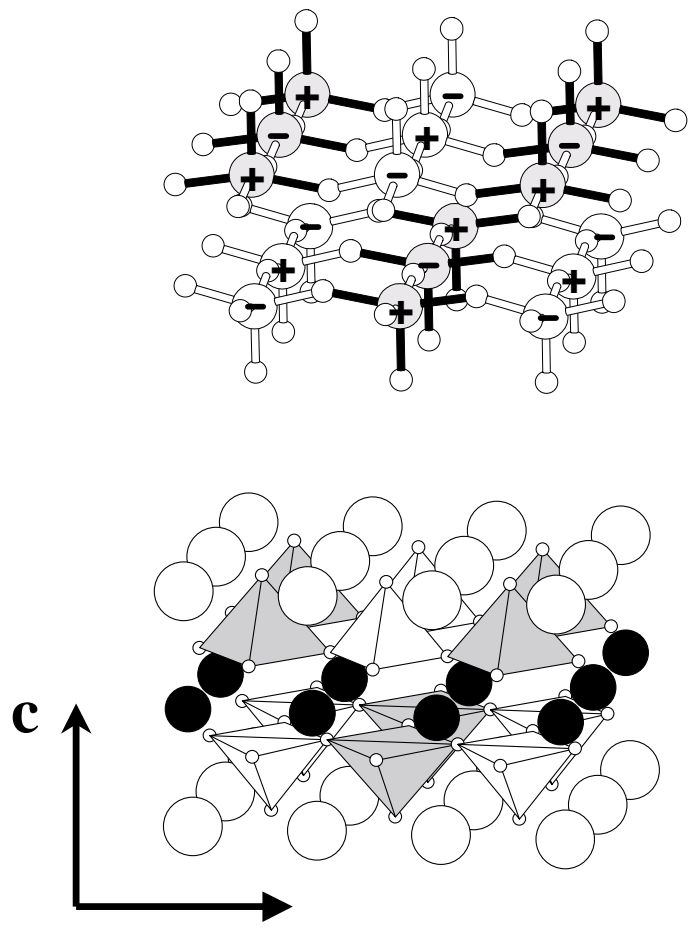

a

FIG. 2. The crystal and magnetic structure of $\mathrm{YBaCo}_{2} \mathrm{O}_{5}$ as determined at $10 \mathrm{~K}$. The top panel shows the individual $\mathrm{Co}^{2+}$ (shaded spheres) and $\mathrm{Co}^{3+}$ (white spheres) ions and their bonds to oxygen (small white spheres). Ba and $\mathrm{Y}$ have been omitted for clarity. All bond lengths $>2.05 \AA$ are drawn in black. The plus and minus symbols denote the antiferromagnetic spin arrangement with the moments aligned parallel to the $b$ axis. The bottom panel shows a polyhedral representation of the structure, highlighting the $\mathrm{Co}^{2+}$ and $\mathrm{Co}^{3+}$ square-pyramidal coordinations. $\mathrm{Ba}$ and $\mathrm{Y}$ ions are represented by white and black spheres, respectively.

see a pronounced upturn in the resistivity, indicative of electron localization, that would be associated with $\mathrm{Co}^{2+} / \mathrm{Co}^{3+}$, charge ordering. This is accompanied by a strong enhancement in the splitting of the $a$ and $b$ axes, which signifies the development of an orbitally ordered state. Using synchrotron $\mathrm{x}$-ray data collected at low temperatures with a PSD, we refined a model of this chargeordered state in order to confirm the charge-ordering phenomenon (see Table II and Fig. 2).

To investigate in more detail the evolution of the magnetic ordering and confirm our observation of charge ordering based on x-ray experiments, we carried out variable temperature neutron powder diffraction experiments. The results of the structural and magnetic refinements are listed in Table II. High-resolution neutron powder diffraction experiments at $400 \mathrm{~K}$ revealed a paramagnetic tetragonal phase. At $300 \mathrm{~K}$ a so-called $G$-type AFM structure [13] is found in which each cobalt ion couples antiferromagnetically to its six neighboring cobalt ions (see Fig. 2). The spins were located in the $a b$ plane with 
TABLE I. Structural models for low-temperature $\mathrm{YBaCo}_{2} \mathrm{O}_{5} \cdot{ }^{a}$

\begin{tabular}{ccc}
\hline \hline Model $^{\mathrm{b}}$ & Space group & Unit cell \\
\hline $\mathrm{a}$ & $P 4 / n b m$ & $\sqrt{2} a \times c$ \\
$\mathrm{~b}$ & $P 4 / n m m$ & $\sqrt{2} a \times c$ \\
$\mathrm{c}$ & $P 4 / m m m$ & $\sqrt{2} a \times c$ \\
$\mathrm{~d}$ & $P m m a$ & $2 a \times b \times c$ \\
\hline \hline
\end{tabular}

aDerived using the program ISOTROPY (written by H. T. Stokes and D. M. Hatch, Department of Physics, Brigham Young University).

${ }^{b}$ For description of each model, see text.

a moment of 2.10(1) $\mu_{B}$ per cobalt ion. This corresponds quite well to the spin-only value expected for a mixed valence compound containing equal concentrations of low spin (LS) $\mathrm{Co}^{2+}\left({ }^{6} t_{2 g}{ }^{1} e_{g}, S=\frac{1}{2}, \mu_{\mathrm{SO}}=1.73 \mu_{B}\right.$ [1]) and intermediate spin (IS) $\mathrm{Co}^{3+}{ }^{3}{ }^{5} t_{2 g} e_{g}, S=1$, $\left.\mu_{\mathrm{SO}}=2.85 \mu_{B}\right)$. The very small orthorhombic splitting prevented the determination of the orientation of the moments within the $a b$ plane.

The refined structural parameters obtained for the low temperature $\mathrm{x}$-ray $(50 \mathrm{~K})$ and neutron $(25 \mathrm{~K})$ data are in good agreement. Bond valence calculations [14] yield valence sums of 2.69 for $\mathrm{Co}(1)$ and 2.02 for $\mathrm{Co}(2)$. These valences and the bond distances confirm the long-range charge ordering of $\mathrm{Co}^{2+} / \mathrm{Co}^{3+}$. However, the charge- ordering topology is not of the $\mathrm{NaCl}$ - or $G$-type as one might expect from purely electrostatic considerations. Instead we have alternating long and short bonds $\left(\mathrm{Co}^{2+}-\mathrm{O}-\mathrm{Co}^{3+}\right)$ along the doubled $a$ axis and chains of either $\mathrm{Co}^{2+}-\mathrm{O}-\mathrm{Co}^{2+}$ or $\mathrm{Co}^{3+}-\mathrm{O}-\mathrm{Co}^{3+}$ linkages running parallel to $b$ (see Fig. 2). The magnetic structure remains $G$-type when charge ordered. However, the magnitude of the spin states is altered because of the ordered distribution of $\mathrm{Co}^{2+}$ and $\mathrm{Co}^{3+}$ ions, which necessarily leads to different magnetic moments. The magnetic structure now contains antiferromagnetic $\mathrm{Co}^{2+}-\mathrm{O}-\mathrm{Co}^{2+}$ and $\mathrm{Co}^{3+}-\mathrm{O}-\mathrm{Co}^{3+}$ chains with the moments aligned parallel to $b$. The $\mathrm{Co}^{3+}$ and $\mathrm{Co}^{2+}$ ions have refined moments of $2.7(1) \mu_{B}$ and $4.2(1) \mu_{B}$, respectively. These values are in good agreement with a charge-ordered state that contains IS $\mathrm{Co}^{3+}\left({ }^{5} t_{2 g}{ }^{1} e_{g}, S=1, \mu_{\mathrm{SO}}=2.85 \mu_{B}\right)$ and HS (high spin) $\mathrm{Co}^{2+}\left({ }^{5} t_{2 g}{ }^{2} e_{g}, S=\frac{3}{2}, \mu_{\mathrm{SO}}=3.87 \mu_{B}\right)$. However, it is conceivable that the observed moments are reduced due to the presence of domains and a model based upon $\mathrm{HS} \mathrm{Co}^{3+}\left({ }^{4} t_{2 g}{ }^{2} e_{g}, S=2, \mu_{\mathrm{SO}}=4.89 \mu_{B}\right)$ and HS $\mathrm{Co}^{2+}\left({ }^{5} t_{2 g}{ }^{2} e_{g}, S=\frac{3}{2}, \mu_{\mathrm{SO}}=3.87 \mu_{B}\right)$ may apply, or as argued in the case of $\mathrm{LaCoO}_{3}$, the ground state could have a strongly mixed character [15]. In any case the experimental data clearly indicate that $\mathrm{YBaCo}_{2} \mathrm{O}_{5}$ undergoes a spin-state transition upon cooling which differs from the one observed in $\mathrm{LaCoO}_{3}$, and the hole-doped

TABLE II. Crystallographic parameters of $\mathrm{YBaCo}_{2} \mathrm{O}_{5}$ obtained from Rietveld refinements.

\begin{tabular}{|c|c|c|c|c|}
\hline $\begin{array}{l}\text { Temp. }(\mathrm{K}) \\
\text { radiation }\end{array}$ & $\begin{array}{c}400 \\
\text { Neutron }\end{array}$ & $\begin{array}{c}300 \\
\text { Neutron }\end{array}$ & $\begin{array}{c}25 \\
\text { Neutron }\end{array}$ & $\begin{array}{c}50 \\
X \text { ray }^{a}\end{array}$ \\
\hline$R_{\mathrm{wp}}{ }^{\mathrm{b}}$ & $6.32 \%$ & $5.78 \%$ & $5.96 \%$ & $10.8 \%$ \\
\hline Space group & $\mathrm{P} 4 / \mathrm{mmm}$ & Pmmm & Pmma & Pmma \\
\hline$a(\AA)$ & $3.89069(2)$ & $3.89219(4)$ & $7.8235(1)$ & $7.83241(6)$ \\
\hline$b(\AA)$ & & $3.88498(4)$ & $3.8746(1)$ & $3.87968(3)$ \\
\hline$c(\AA)$ & $7.4994(1)$ & $7.4808(1)$ & $7.4367(2)$ & $7.44394(5)$ \\
\hline \multicolumn{5}{|l|}{ Atomic positions } \\
\hline $\mathrm{Ba}$ & $0,0,0$ & $\frac{1}{2}, \frac{1}{2}, 0$ & $0,0,0$ & $0,0,0$ \\
\hline $\mathrm{Y}$ & $0,0, \frac{1}{2}$ & $\frac{1}{2}, \frac{1}{2}, \frac{1}{2}$ & $0,0, \frac{1}{2}$ & $0,0, \frac{1}{2}$ \\
\hline $\operatorname{Co}(1)$ & $\frac{1}{2}, \frac{1}{2}, 0.2593(4)$ & $0,0,0.2585(4)$ & $\frac{1}{4}, \frac{1}{2}, 0.271(2)$ & $\frac{1}{4}, \frac{1}{2}, 0.2687(5)$ \\
\hline $\operatorname{Co}(2)$ & $\ldots$ & $\cdots$ & $\frac{1}{4}, \frac{1}{2}, 0.755(2)$ & $\frac{1}{4}, \frac{1}{2}, 0.7509(5)$ \\
\hline $\mathrm{O}(1)$ & $\frac{1}{2}, \frac{1}{2}, 0$ & $0,0,0$ & $\frac{1}{4}, \frac{1}{2},-0.006(1)$ & $\frac{1}{4}, \frac{1}{2}, 0.000(4)$ \\
\hline $\mathrm{O}(2)$ & $0, \frac{1}{2}, 0.3122(1)$ & $0, \frac{1}{2}, 0.3140(9)$ & $\frac{1}{4}, 0,-0.317(1)$ & $\frac{1}{4}, 0,-0.309(3)$ \\
\hline $\mathrm{O}(3)$ & $\ldots$ & $\frac{1}{2}, 0,0.3096(9)$ & $\frac{1}{4}, 0,0.307(1)$ & $\frac{1}{4}, 0,0.310(3)$ \\
\hline $\mathrm{O}(4)$ & $\cdots$ & $\ldots$ & $-0.0153(5), \frac{1}{2}, 0.3105(5)$ & $-0.014(2), \frac{1}{2}, 0.3135(9)$ \\
\hline \multirow[t]{3}{*}{ Co(1)-O Dist. } & $1 \times 1.944(3)$ & $1 \times 1.934(3)$ & $1 \times 2.06(2)$ & $1 \times 2.00(3)$ \\
\hline & $4 \times 1.9853(7)$ & $2 \times 1.986(2)$ & $2 \times 1.955(2)$ & $2 \times 1.964(4)$ \\
\hline & & $2 \times 1.983(1)$ & $2 \times 2.096(5)$ & $2 \times 2.08(2)$ \\
\hline \multirow{3}{*}{ Co(2)-O Dist. } & & & $1 \times 1.82(2)$ & $1 \times 1.87(3)$ \\
\hline & & & $2 \times 2.004(4)$ & $2 \times 1.991(6)$ \\
\hline & & & $2 \times 1.894(5)$ & $2 \times 1.93(2)$ \\
\hline
\end{tabular}

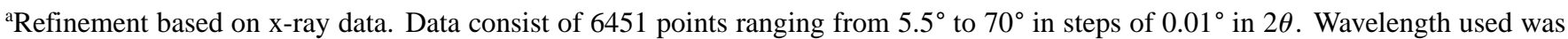
$0.79891 \AA$ determined by a calibration with a $\mathrm{CeO}_{2}$ standard.

${ }^{\mathrm{b}} R_{\mathrm{wp}}=\left\{\sum w_{i}\left[\left(y_{i}\right)_{\mathrm{obs}}-\left(y_{i}\right)_{\mathrm{calc}}\right]^{2} / \sum w_{i}\left[\left(y_{i}\right)_{\mathrm{obs}}\right]^{2}\right\}^{1 / 2} ; y_{i}$ is the background-corrected intensity at point $i$. The weights $w_{i}$ are derived purely from counting statistics. The summation is taken over all points that contribute to Bragg intensities. 
systems $\mathrm{La}_{1-x} \mathrm{Sr}_{x} \mathrm{CoO}_{3}$, in three important aspects: (i) the spin-state transition occurs within a long-range AFM ordered magnetic structure, (ii) it is driven by long-range charge ordering, and (iii) the spin state changes from low spin to high spin upon cooling [16].

We point out that neutron powder diffraction measurements on similar earlier samples produced results different from those reported above. The pronounced splitting of the $a$ and $b$ axes at low temperatures was not observed, suggesting the absence of long-range charge and orbital ordering. Furthermore, the refined magnetic moment at $12 \mathrm{~K}$ was determined to be $2.16(2) \mu_{B}$ per cobalt, very similar to the value obtained at $300 \mathrm{~K}$, thus indicating that no spin-state transition occurred. The absence of both charge ordering and spin-state transition can be attributed to an increased oxygen content of these samples. In our initial experiments we did not take sufficient care to prevent room temperature oxidation of the material. The subsequent change in the $\mathrm{Co}^{2+}: \mathrm{Co}^{3+}$ ratio destabilizes the charge-ordered state and the spin-state transition as well. This extreme sensitivity of the charge-orderinduced spin-state transition to the oxygen content helps explain the above mentioned phase coexistence occurring at $T_{\mathrm{N}}$. It appears plausible that certain regions of the sample, most likely the surface, can have an oxygen stoichiometry slightly in excess of 5.00. These regions would remain in the tetragonal state without undergoing charge ordering and the subsequent spin-state transition, as observed in bulk samples with defined oxygen stoichiometries greater than 5.00. This is corroborated by the refined magnetic moment of 2.5(1) $\mu_{B}$ for the mixed valent cobalt in the tetragonal minority tetragonal phase at $25 \mathrm{~K}$.

In summary, we have demonstrated that the low temperature structural phase transition in $\mathrm{YBaCo}_{2} \mathrm{O}_{5}$ is a spinstate transition driven by a charge and orbital ordering which occurs in a $G$-type AFM structure. The charge ordering topology of $\mathrm{Co}^{2+}$ and $\mathrm{Co}^{3+}$ is distinct from that observed in the isostructural manganates where $T_{\mathrm{CO}} \geq T_{\mathrm{N}}$ [12]. Furthermore, it has been shown that the incorporation of excess oxygen into the rare earth layer destroys the charge-ordering and spin-state transition, thus confirming the strong coupling between the two. The change from a LS to a HS state upon cooling, as well as the presence of AFM spin ordering, is distinctly different from the behavior observed in $\mathrm{LaCoO}_{3}$ and $\mathrm{La}_{1-x} \mathrm{Sr}_{x} \mathrm{CoO}_{3}$. To our knowledge, $\mathrm{YBaCo}_{2} \mathrm{O}_{5}$ is the first material to exhibit a spin-state transition in combination with long-range charge, spin, and orbital ordering.

The authors thank D.E. Cox, M. Strongin (BNL), and B. J. Kennedy (Sydney University) for stimulating discussions. T. V. thanks the Australian Nuclear Science \& Technology Organization and, in particular, the members of the Neutron Scattering Group at Lucas Heights for their hospitality during various visits. Research at the National Synchrotron Light Source at Brookhaven National Laboratory was carried out under Contract No. DE-AC0298CH10886, Division of Materials Sciences, U.S. Department of Energy.

[1] R. R. Heikes, R. C. Miller, and R. Mazelsky, Physica (Utrecht) 30, 1600 (1964).

[2] G. H. Jonker, J. Appl. Phys. 37, 1424 (1966).

[3] K. Asai, A. Yoneda, O. Yokokura, J. M. Tranquada, G. Shirane, and K. Kohn, J. Phys. Soc. Jpn. 67, 290 (1998).

[4] M. A. Señarís-Rodríguez and J. B. Goodenough, J. Solid State Chem. 118, 323 (1995).

[5] A. Mahendiran and A. K. Raychaudhuri, Phys. Rev. B 54, 16044 (1996).

[6] C. Martin, A. Maignan, D. Pelloquin, N. Nguyen, and B. Raveau, Appl. Phys. Lett. 71, 1421 (1997).

[7] A. Maignan, C. Martin, D. Pelloquin, N. Nguyen, and B. Raveau, J. Solid State Chem. 142, 247 (1999).

[8] Synchrotron X-ray diffraction data were collected at X7A at the NSLS at Brookhaven National Laboratory using a $\mathrm{Ge}(111)$ monochromator. Samples were loaded into $0.2 \mathrm{~mm}$ capillaries. Data were collected either using a linear PSD [G. Smith, Synchrotron Radiation News 4, 24 (1991)] or a single scintillation detector (Bicron) and a $\mathrm{Si}(111)$ as analyzer.

[9] Neutron powder diffraction data were collected using high resolution powder diffractometer [C. J. Howard, C. J. Ball, R. L. Davis, and M. M. Elcombe, Aust. J. Phys. 36, 507 (1983)] located at the HIFAR research reactor operated by the Australian Nuclear Science \& Technology Organization at Lucas Heights, Australia.

[10] P. Karen and A. Kjekshus, J. Am. Ceram. Soc. 77, 547 (1994).

[11] P. M. Woodward, D.E. Cox, T. Vogt, C. N. R. Rao, and A. K. Cheetham, Chem. Mater. 11, 3528-3538 (1999); P. M. Woodward, D. E. Cox, and T. Vogt, J. Supercond. (to be published).

[12] F. Millange, E. Suard, V. Caignaert, and B. Raveau, Mater. Res. Bull. 34, 1 (1999).

[13] E. O. Wollan and W.C. Koehler, Phys. Rev. 100, 545 (1955).

[14] M. O'Keeffe, "Eutax. Program for Calculating Bond Valences," EMLab, Phoenix, AZ.

[15] A. Chainani, M. Mathew, and D. D. Sarma, Phys. Rev. B 46, 9976 (1992).

[16] Subsequent to our initial submission of this Letter, E. Suard et al. reported antiferromagnetic and charge-ordering transitions in $\mathrm{HoBaCo}_{2} \mathrm{O}_{5}$, which appear to be in complete agreement with our findings in $\mathrm{YBaCo}_{2} \mathrm{O}_{5}$, at the XVIIth IUCr Congress in Glasgow (1999). However, no spin-state transition was reported. 